\title{
Ball Mill Automatic Control System Design Based on Particle Swarm Optimization Algorithm
}

\author{
$\mathrm{Li} \mathrm{Ai}$ and Yan Xiong \\ The Engineering College of Honghe University, Mengzi, 661199, China \\ 364508229@qq.com, cjt@163.com
}

\begin{abstract}
Keywords: Ball mill; Particle swarm optimization (PSO); Neural network; PID control; Constant power
\end{abstract}

\begin{abstract}
For ball mill grinding process random interference by many factors, processes complex mechanism, there was a big inertia and lag, conventional PID control effect was poor, the particle swarm optimization neural network approach was introduced into the mill control system, it had strong robustness, can effectively overcome the mill main motor power nonlinear, time-varying factors such as interference. System was reliable, adjust speed, anti-interference ability, can better achieve constant power automatic control of the ball mill, with good application value.
\end{abstract}

\section{Introduction}

Ball is the material to be broken, and then to smash the key equipment, has a simple structure, large capacity single, crushing ratio, yield, continuous operation and stable, becomes major grinding equipment [1]. In the grinding process, it should strictly control the ball, mine and water supply for a ball mill, these parameters have a significant impact on product quality.

Because the system inertia and there is a big lag, and also by external feeding conditions, when the nature of the material entering the mill changed, resulting in a large ball mill load fluctuations, the performance of operating current to swing, and the only experience to discriminate swelling grinding mill is in operation or empty mill state, likely to cause damage to the ball mill, motors and transmission machinery affecting the normal operation, the product quality can't be guaranteed, so its control is necessary and achieve its automatic control has broad application prospects [2].

As a parameter optimization problem, PID controller design is to find the optimal value in the solution space, so as to achieve the purpose of tracking error is minimized. Therefore, how to carry out parameter optimization has become a hot spot direction for PID controller design and applications. With the development of artificial intelligence, the researchers made a series of related methods to optimize PID controller parameters, such as genetic algorithms (GA) [3], particle swarm optimization (PSO) [4,5] and ant colony optimization (ACO ) [6]. However, the genetic algorithm need to replicate, crossover and mutation, and easy to produce premature convergence [7]. The programming of ant colony algorithm was relatively complicated, versatility was not high, limiting its scope of application. The particle swarm algorithm was simple, fast convergence, this paper used PSO algorithm to optimize PID controller parameters.

\section{PID Parameter Optimization Realization}

Particle Swarm Optimization. In the d-dimensional search space, there is a group of $m$ particles, each particle $\mathrm{i}(\mathrm{i}=1,2, \ldots, \mathrm{m})$ contains a velocity vector $\mathrm{v}_{\mathrm{i}}=\mathrm{v}_{\mathrm{i} 1}, \mathrm{v}_{\mathrm{i} 2}, \ldots, \mathrm{v}_{\mathrm{id}}$ and position vector $x_{i}=\left(x_{i 1}, x_{i 2}, \ldots, x_{i d}\right)$. The particle experiences the optimal location denoted $p_{i}=\left(p_{i 1}, p_{i 2}, \ldots, p_{i d}\right)$, and the particle swarm best position experienced denoted $p_{g}=\left(p_{g 1}, p_{g 2}, \ldots, p_{g d}\right)$. In each iteration, particle $\mathrm{i}$ by tracking the two optimal values, in accordance with the following formula updates velocity and position.

$$
\begin{aligned}
& v_{i d}(k+1)=w v_{i d}(k)+c_{1} r_{1}\left(p_{i d}(k)-x_{i d}(k)+c_{2} r_{2}\left(p_{g d}(k)-x_{i d}(k)\right)\right. \\
& x_{i d}(k+1)=x_{i d}(k)+v_{i d}(k+1)
\end{aligned}
$$


Which, $v_{i d}$ is the particle velocity vector; $x_{i d}$ is the particle position vector; $\mathrm{w}$ for the inertia weight, which allows particles to maintain inertia; $c_{1}, c_{2}$ for the learning factors, general $\mathrm{c}_{1}=\mathrm{c}_{2}=2 ; \mathrm{k}$ is the iteration number; $\mathrm{r}_{1}, \mathrm{r}_{2}$ for random numbers between 0 and 1 . In addition, particles adjust their position, and limited by the maximum speed $\mathrm{v}_{\max }$.

In order to balance the particle swarm global and local search capabilities, inertia weight often use Eq. 3 to adaptive adjustment.

$$
w=w_{\text {start }}-\frac{w_{\text {start }}-w_{\text {end }}}{k_{\max }} \times k
$$

Where, $\mathrm{k}_{\max }$ represents the maximum number of iterations, $\mathrm{w}_{\text {start }}, \mathrm{w}_{\mathrm{end}}$, respectively, the initial inertia weight and end inertia weight, $\mathrm{k}$ represents the current iteration number [8].

BP network was one of the most widely used neural networks, its learning and training process was composed of the forward propagation and backward propagation. These two modes of transmission were carried out again and again, until the network error sum of squares to achieve the desired accuracy. Because of its algorithm was based on gradient descent, with easy to fall into local optimum, slow convergence and other shortcomings.

Particle swarm optimization neural network algorithm was the particle in its search space search weights and thresholds between layers. First, give the BP network weights, threshold $[0,1]$ random number. Secondly, according to the BP network topology calculate the output value. Then, using the adaptive mutation particle swarm algorithm search out the best position to make the fitness function to a minimum.

Control system main control objective was to maintain the stability of ball mill load, which was to stabilize the power of the control drive motor [9]. Affect the drive motor power stability was a major factor mill feeding amount and deposit size. The deposit size was generally set value, the floating range was small; the system was mainly used to control the feed quantity to control the motor in constant power state. Ball automatic control system structure was shown in Fig. 1.

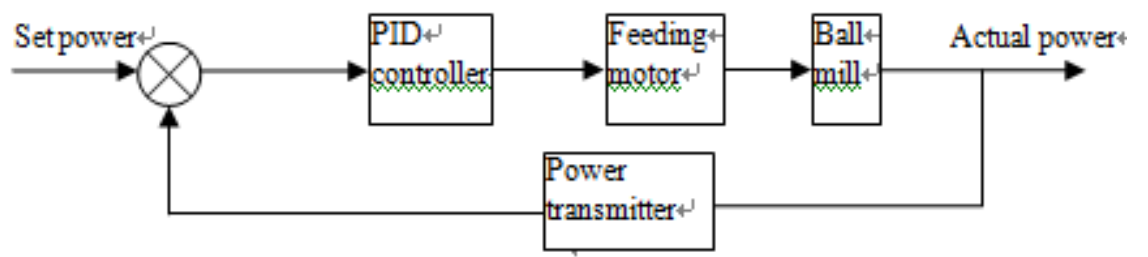

Figure 1. Ball automatic control system structure

Main drive motor power detected by the power transmitter, the detected power signal was converted into an electrical signal sent to the PSO PID controller, the PID controller using four inputs and three outputs, the detected power value and set the power of comparison, the error value $\mathrm{e}$, voice, vibration and temperature as the PID controller inputs, output KP, TI, TD PID control parameters, according to the parameters of the controlled process for KP, TI, TD's self-tuning parameters required to achieve KP, TI, TD-tuning to meet the different moments motor power, so that the actual controller had good dynamic performance, PID of the three parameters can be based on the current system status to make the appropriate adjustments.

\section{Hardware Design}

System selected Siemens S7-400 type manufactured PLC as controller, the hardware block diagram shown in Fig. 2. The whole control system PLC as the core controller, through the voice of the transmitter detected a ball mill grinding sound, vibration acceleration transmitter detected the spindle vibration, temperature transmitter detected the temperature of lubricating oil, pressure transmitter detected pressure of lubricating oil, power transmitter detected a power drive motor controller according to collected data, the use of PSO control model output a certain amount of 
control to the mine through the inverter to adjust the motor speed to achieve constant main electrical power, that was ball workload stability control objectives, System design as a field of human-computer touch-screen interface for communication via PROFIBUS and remote monitoring host computer to communicate in order to monitoring and controlling of the work state of the mill in the control room, improved the mill control system automation[10].

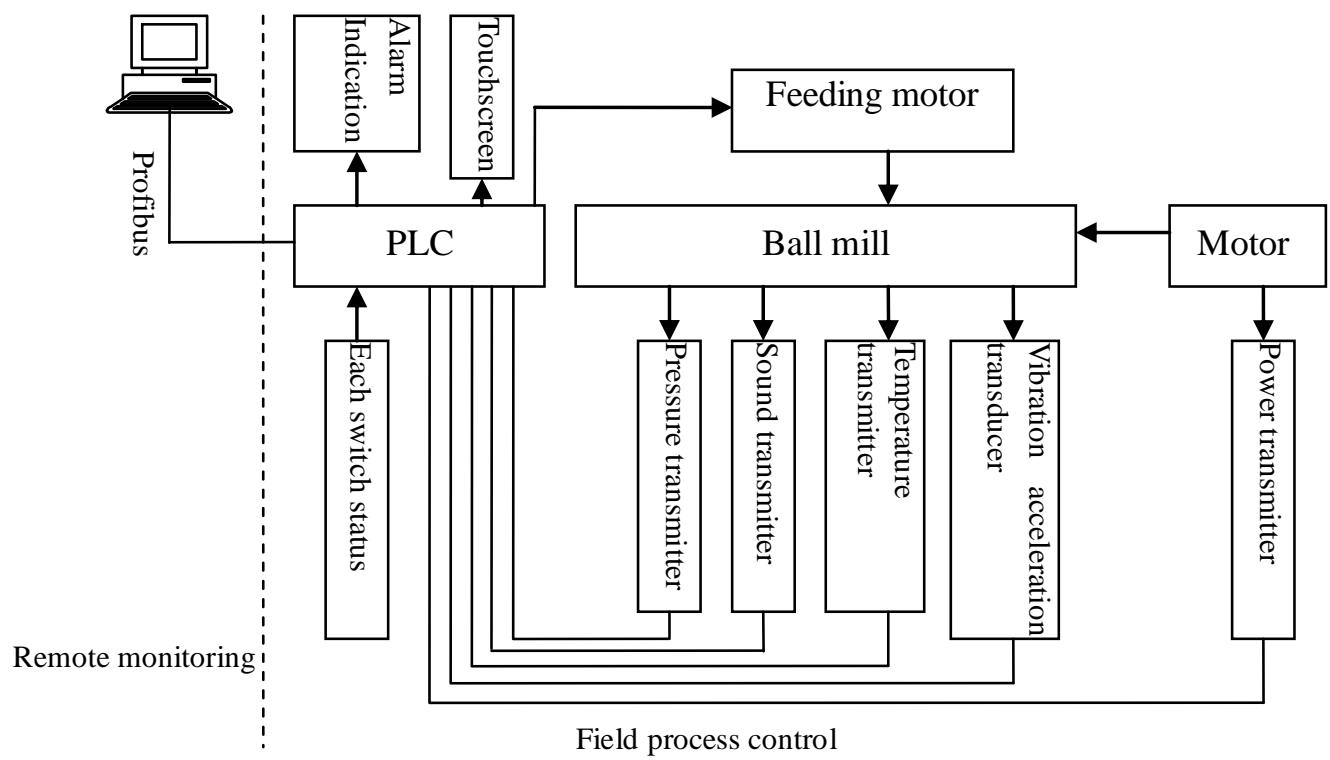

Figure 2. Hardware block diagram

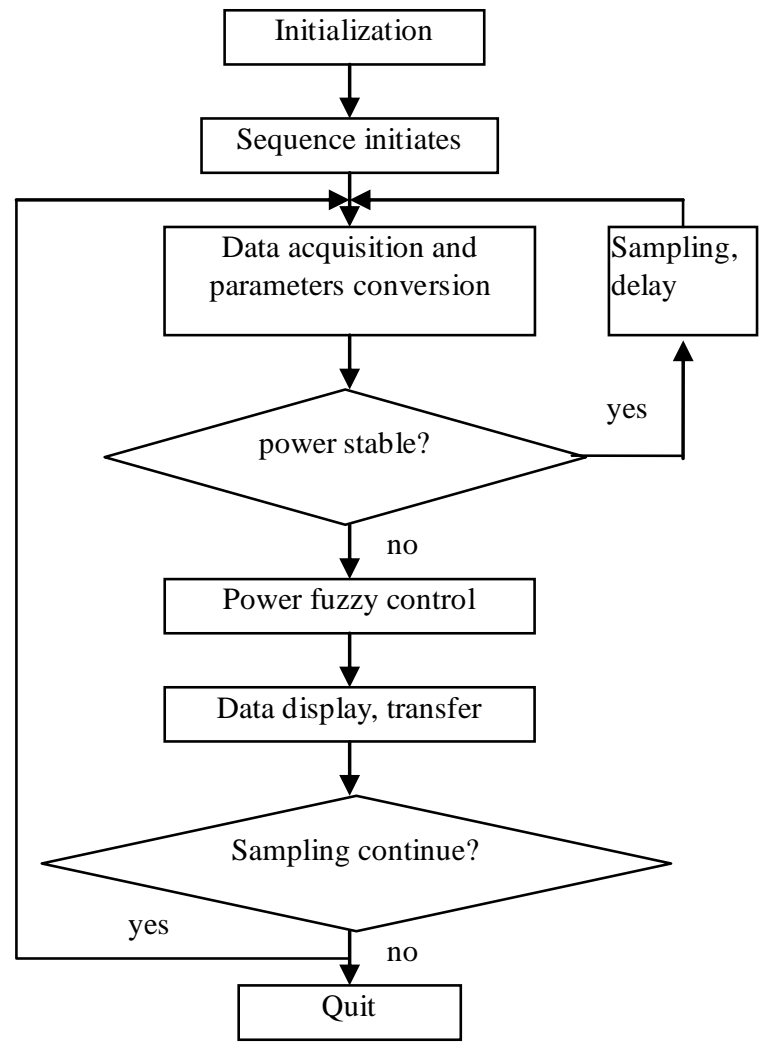

Figure 3. System control software flow chart

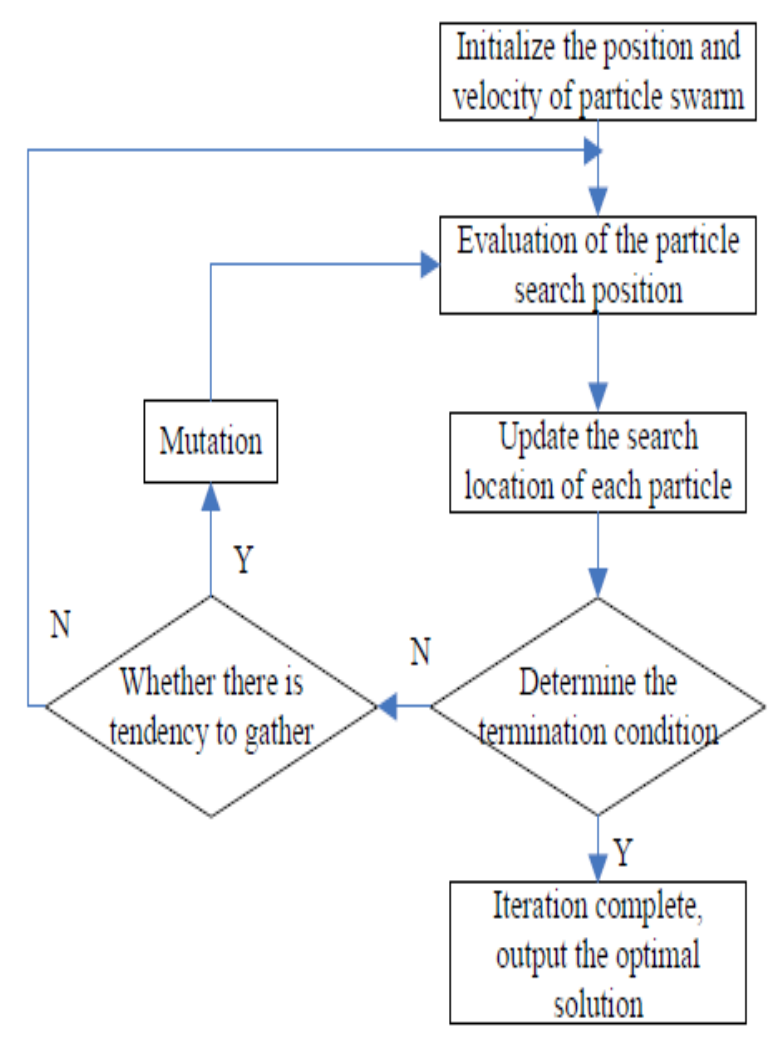

Figure 4. PSO control algorithm flow chart 


\section{System Software Design}

The main control program written using STEP7 software debugging, the control system program included initialization, data collection and scale conversion, PSO power adjustment, the system control software flow chart shown in Fig. 3, PSO control algorithm flow chart shown in Fig. 4.

\section{Conclusion}

For ball mill grinding process random interference by many factors, processes mechanisms were complicated, there was a big inertia and lag, the PSO algorithm was introduced into the mill control system, it had strong robustness, can effectively overcome the mill main motor power nonlinear, time-varying and other factors interference. System was reliable, adjust speed, anti-interference ability, can better achieve constant power automatic control of the ball mill, with good application value.

\section{References}

[1] TAN Lu-min, FENG Xin-gang, Design of Automatic Control System of Ball Mill Based on Fuzzy PID Control, Coal Mine Machinery, 33(2) (2012) 170-172.

[2] Dang Hong-she, Zhang Ying, Wang Gang, Ball Mill Control System Design, Process Automation Instrumentation, 04 (2014) 26-29.

[3] MENG An-bo, YIN Hao, Multi-Agent genetic algorithm for PID governor, Electric Power Automation Equipment, 32(9) (2012) 128-133.

[4] WeiDer Chang, ShunPeng Shin, PID controller design of nonlinear systems using an improved particle swarm optimization approach, Communication in Nonlinear Science and Numerical Simulation, 5(11) (2010) 3632-3639.

[5] GUO Wei, HAN Dan-dan, XU Jin-cheng, et al, Fractional Order PID Predictive Functional Control Parameter Tuning Based on Particle Swarm Optimization, Control Engineering of China, 21(1) (2014) 70-73.

[6] VAROL H A,BINGUL Z, A new PID tuning technique using ant algorithm, Proceedings of American Control Conference, Boston, USA, IEEE, 2004, 2154-2159.

[7] KOU Pan-gao, ZHOU Jian-zhong, HE Yao-yao, et al, Optimal PID Governor Tuning of Hydraulic Turbine Generators With Bacterial Foraging Particle Swarm Optimization Algorithm, Proceedings of the CSEE, 29(26) (2009) 101-106.

[8] Tang Yu-lan, Xu Ming-liang, Mei Juan, et al, Particle Swarm Optimization Research and Application in PID Controller parameters tuning, Computer Engineering and Applications, 48(34) (2012) 221-225.

[9] Man Hong, Liang Ying-chun, Ji Yong-gang, et al, Principles of Automatic Control, Tsinghua University Press, Beijing, 2011.

[10]Zhao Fang-ting, Chen Shu-liang, VC-based OPC communication between PC and PLC, Information \& Communications, 8(2015) 46-47. 